\title{
Author Correction: Resonant tunnelling diodes based on twisted black phosphorus homostructures
}

Pawan Kumar Srivastava (D), Yasir Hassan, Duarte J. P. de Sousa, Yisehak Gebredingle, Minwoong Joe (D), Fida Ali (D), Yang Zheng, Won Jong Yoo (iD), Subhasis Ghosh, James T. Teherani D, Budhi Singh (D), Tony Low and Changgu Lee $\mathbb{D}$

Correction to: Nature Electronics https://doi.org/10.1038/s41928-021-00549-1, published online 8 March 2021.

In the version of this Article originally published, in the Acknowledgements, the project number relating to the Samsung Research Funding Center of Samsung Electronics was incorrectly given as "SRFC- MA1802-01"; it should have been 'SRFC-TB1803-04'. All versions of the Article have been amended.

Published online: 9 July 2021

https://doi.org/10.1038/s41928-021-00621-w

(c) The Author(s), under exclusive licence to Springer Nature Limited 2021 